\title{
PERSPECTIVA DEL DERECHO DEL MEDIO AMBIENTE Y DE LAS POLÍTICAS AMBIENTALES DE LA UNIÓN EUROPEA
}

\author{
JORGE AGUDO GONZÁLEZ \\ Profesor titular de Derecho Administrativo \\ Universidad Autónoma de Madrid \\ LORENA TRUJILLO PARRA \\ Becaria de investigación \\ Universidad Autónoma de Madrid
}


Sumario: 1. Introducción. 2. Actividad de las instituciones en el ámbito de la política europea de medio ambiente. 2.1. Actos conjuntos del Consejo y del Parlamento Europeo. A. Reglamento n. ${ }^{\circ} 1152 / 2012$, del Parlamento Europeo y del Consejo, de 21 de noviembre de 2012, por el que se modifica el Reglamento (CE) n..$^{\circ}$ 2371/2002 del Consejo, sobre la conservación y la explotación sostenible de los recursos pesqueros en virtud de la política pesquera común. B. Reglamento n. ${ }^{\circ}$ 1026/2012 del Parlamento Europeo y del Consejo, de 25 de octubre de 2013, sobre determinadas medidas destinadas a la conservación de las poblaciones de peces en relación con los países que autorizan una pesca no sostenible. 2.2. Actos del Consejo. A. Reglamento n. ${ }^{\circ} 1243 / 2012$ del Consejo, de 19 de diciembre de 2012, que modifica el Reglamento (CE) n. ${ }^{\circ} 1342 / 2008$ por el que se establece un plan a largo plazo para las poblaciones de bacalao y las pesquerías que las explotan. 2.3. Actos del Parlamento. A. Resolución del Parlamento Europeo, de 29 de septiembre de 2011, sobre la elaboración de una posición común de la UE ante la Conferencia de las Naciones Unidas sobre el Desarrollo Sostenible (Río+20) (2013/C 56 E/14). B. Resolución del Parlamento Europeo, de 14 de septiembre de 2011, sobre un enfoque global con respecto a las emisiones antropogénicas de gases distintos al $\mathrm{CO}_{2}$ que afectan al clima (2013/C 51 E/14). C. Resolución del Parlamento Europeo, de 11 de mayo de 2011, sobre el Libro Verde de la Comisión sobre protección de los bosques e información forestal en la UE: Preparación de los bosques al cambio climático (2010/2106(INI)) (2012/C 377 E/05). 2.4. Actos de la Comisión. A. Reglamento n. ${ }^{\circ} 1158 / 2012$ de la Comisión, de 27 de noviembre de 2012, que modifica el Reglamento (CE) n. ${ }^{\circ}$ 338/97 del Consejo, relativo a la protección de especies de la fauna y flora silvestres mediante el control de su comercio. B. Directiva 2012/40/UE de la Comisión, de 26 de noviembre de 2012, por la que se corrige el anexo I de la Directiva 98/8/CE del Parlamento Europeo y del Consejo, relativa a la comercialización de biocidas. C. Dictamen de la Comisión, de 18 de diciembre de 2012, sobre el plan de eliminación de los residuos radiactivos producto del desmantelamiento de la central nuclear de KLW, situada en Lingen, Baja Sajonia, Alemania. D. Decisión de Ejecución de la Comisión, de 19 de febrero de 2013, relativa a las limitaciones a las autorizaciones de biocidas que contengan difenácum notificadas por Alemania de conformidad con la Directiva 98/8/CE del Parlamento Europeo y del Consejo (2013/96/UE). E. Decisión de la Comisión, de 13 de diciembre de 2012, relativa a las disposiciones nacionales notificadas por Austria sobre determinados gases industriales de efecto invernadero (2012/784/UE). F. Decisión de la Comisión, de 14 de noviembre de 2012, por la que se cursa una notificación a los terceros países que la Comisión estima susceptibles de ser considerados terceros países no cooperantes conforme al Reglamento (CE) n. ${ }^{\circ} 1005 / 2008$ del Consejo por el que se establece un sistema comunitario para prevenir, desalentar y eliminar la pesca ilegal, no declarada y no reglamentada (2012/C 354/01). G. Decisión de Ejecución de la Comisión, de 23 de octubre de 2012, por la que se concede la exención solicitada por el Reino de Dinamarca de conformidad con la Directiva 91/676/CEE del Consejo, relativa a la protección de las aguas contra la contaminación producida por nitratos utilizados en la agricultura (2012/659/UE). H. Decisión de Ejecución de la Comisión, de 23 de noviembre de 2012, sobre el reconocimiento del régimen "Roundtable on Sustainable Palm Oil RED" para demostrar el cumplimiento de los criterios de sostenibilidad con las directivas 98/70/CE y 2009/28/CE del Parlamento Europeo y del Consejo (2012/722/UE). I. Decisión de Ejecución de la Comisión, de 14 de noviembre de 2012, por la que se establece un formato común para la presentación de la información prevista en la Directiva 2010/63/UE del Parlamento Europeo y del Consejo, relativa a la protección de los animales utilizados para fines científicos (2012/707/UE). 2.5. Otros. A. Anuncio de la Comisión Europea a las empresas que tengan la intención de importar a la Unión, o exportar desde esta, sustancias reguladas que agotan la capa de ozono en 2014 y a las empresas que tengan la intención de producir o importar esas sustancias para usos esenciales de laboratorio y análisis en 2014 (2013/C 25/06). B. Dictamen del Comité de las Regiones: La innovación al servicio del crecimiento sostenible: una bioeconomía para Europa (2013/C 17/09). C. Dictamen del Comité Económico y Social Europeo sobre la "Comunicación de la Comisión al Parlamento Europeo, al Consejo, al Comité Económico y Social Europeo y al Comité de las Regiones — Innovación para un futuro sostenible. Plan de Acción sobre Ecoinnovación (Eco-AP)" [COM (2011) 899 final], (2012/ C 351/14). D. Dictamen del Comité Económico y Social Europeo sobre la "Propuesta de Reglamento del Parlamento Europeo y del Consejo, por el que se modifica el Reglamento (CE) n. ${ }^{\circ} 850 / 98$ del Consejo para la conservación de los recursos pesqueros a través de medidas técnicas de protección de los juveniles de organismos marinos y se deroga el Reglamento (CE) n. ${ }^{\text {o }}$ 1288/2009 del Consejo" [COM(2012) 298 final — 2012/0158 (COD)] (2012/C 351/18). E. Dictamen del Comité Económico y Social Europeo sobre la "Propuesta de Reglamento del Parlamento Europeo y del Consejo, que modifica el Reglamento (CE) n. ${ }^{\circ}$ 1005/2008 del Consejo, por el que se establece un sistema comunitario para prevenir, desalentar y eliminar la pesca ilegal, no declarada y no reglamentada" [COM(2012) 332 final 2012/162 (COD)], (2012/C 351/21). F. Dictamen del Comité de las Regiones - Fondo Europeo Marítimo y de Pesca (FEMP) (2012/C 391/10). G. Dictamen del Comité Económico y Social Europeo sobre la "Propuesta de Reglamento del Parlamento Europeo y del Consejo que modifica el Reglamento 
(CE) n. ${ }^{\circ}$ 2187/2005 relativo a la conservación, mediante medidas técnicas, de los recursos pesqueros en aguas del mar Báltico, los Belts y el Sund" [COM (2012) 591 final - 2012/0285 (COD)] (2013/C 44/28). H. Dictamen del Comité Económico y Social Europeo sobre la "Comunicación de la Comisión al Parlamento Europeo, al Consejo, al Comité Económico y Social Europeo y al Comité de las Regiones relativa a la Cooperación de Innovación Europea sobre el Agua" [COM (2012) 216 final] (2013/C 44/26). I. Dictamen del Comité Económico y Social Europeo sobre la "Comunicación de la Comisión al Consejo y al Parlamento Europeo sobre las evaluaciones completas del riesgo y de la seguridad ('pruebas de resistencia') de las centrales nucleares de la Unión Europea y actividades relacionadas" [COM (2012) 571 final]. (2013/C 44/25). J. Dictamen del Comité Económico y Social Europeo sobre la "Propuesta de Reglamento del Parlamento Europeo y del Consejo que modifica el Reglamento (CE) n. ${ }^{\circ}$ 443/2009 a fin de definir las normas para alcanzar el objetivo de 2020 de reducción de las emisiones de $\mathrm{CO}_{2}$ de los turismos nuevos [COM (2012) 393 final - 2012/0190 (COD)] en virtud de la Propuesta de Reglamento del Parlamento Europeo y del Consejo por el que se modifica el Reglamento (UE) n. ${ }^{\circ} 510 / 2011$ a fin de establecer las normas para alcanzar el objetivo de 2020 de reducción de las emisiones de $\mathrm{CO}_{2}$ de los vehículos comerciales ligeros nuevos [COM (2012) 394 final - 2012/0191 (COD)], (2013/C 44/19). K. Dictamen del Comité Económico y Social Europeo sobre "Río+20: balance y perspectivas" (Dictamen adicional) (2013/C 44/11). L. Dictamen del Comité Económico y Social Europeo sobre la "Propuesta de Decisión del Parlamento Europeo y del Consejo, sobre las normas contables y los planes de acción aplicables a las emisiones y absorciones de gases de efecto invernadero resultantes de las actividades enmarcadas en el uso de la tierra, el cambio de uso de la tierra y la silvicultura" [COM (2012) 93 final 2012/0042 (COD), (2012/C 351/19)]. M. Dictamen del Comité Económico y Social Europeo sobre la Comunicación de la Comisión al Parlamento Europeo, al Consejo, al Comité Económico y Social Europeo y al Comité de las Regiones "Energías renovables: principales protagonistas en el mercado europeo de la energía" [COM(2012) 271 final], (2013/C 44/24).

\section{Introducción}

Las instituciones de la Unión Europea han realizado una intensa actividad en el marco de la política medioambiental comunitaria en el período transcurrido desde finales del último trimestre de 2012 hasta mediados del mes de marzo de 2013. La exposición se ordena en función de la institución emisora del acto jurídico, y dentro de la actividad de cada institución, en la medida de lo posible se ordena temáticamente.

\section{Actividad de las instituciones en el ámbito de la política europea de medio ambiente}

\subsection{Actos conjuntos del Consejo y del Parlamento Europeo}

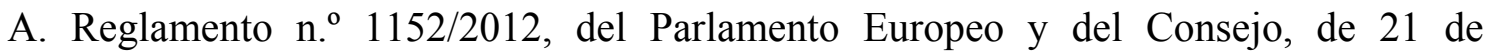
noviembre de 2012, por el que se modifica el Reglamento (CE) n. ${ }^{\circ}$ 2371/2002 del Consejo, sobre la conservación y la explotación sostenible de los recursos pesqueros en virtud de la política pesquera común

El Reglamento prevé que, desde el 1 de enero de 2013 hasta el 31 de diciembre de 2014, se podrá autorizar a los Estados miembros, en las aguas comprendidas hasta las 12 millas marinas desde las líneas de base que estén sometidas a su jurisdicción, a 
restringir la pesca a los buques pesqueros que tradicionalmente faenen en esas aguas y procedan de puertos situados en la costa cercana. Todo ello sin perjuicio de las disposiciones aplicables a los buques pesqueros de la Unión que enarbolen el pabellón de otros Estados miembros en virtud de las relaciones de vecindad existentes entre los Estados miembros, del mismo modo que de las disposiciones del anexo I del Reglamento, en el que se fijan, para cada Estado miembro, las zonas geográficas situadas dentro de las aguas costeras de los demás Estados miembros en que se realizan actividades pesqueras, así como las especies afectadas.

B. Reglamento n. ${ }^{\text {1 }}$ 1026/2012 del Parlamento Europeo y del Consejo, de 25 de octubre de 2013, sobre determinadas medidas destinadas a la conservación de las poblaciones de peces en relación con los países que autorizan una pesca no sostenible

El citado Reglamento establece un marco para la adopción de determinadas medidas relativas a las actividades y las políticas de terceros países relacionadas con la pesca, con el fin de garantizar la conservación a largo plazo de las poblaciones de peces de interés común para la Unión y esos terceros países. Asimismo, establece diversas medidas dirigidas a aquellos países que permiten una pesca no sostenible. Tal y como establece el artículo 4 del Reglamento, estas medidas prevén que la Comisión podrá adoptar actos de ejecución en los que se impongan restricciones cuantitativas a las importaciones de ejemplares de interés general o asociados con esas especies. Otras medidas que establece el Reglamento son que la Comisión podrá imponer restricciones a la utilización de puertos de la Unión por parte de buques con pabellón de tales países (aquellos que permiten una pesca no sostenible) que pesquen la población de peces de interés común o las especies asociadas, y prohibir la compra por parte de los operadores económicos de la Unión de buques pesqueros con pabellón de esos países, entre otras.

El Reglamento prevé un procedimiento para la adopción de estas medidas en sus artículos 6 y siguientes, del que se ha de destacar la asistencia de un comité en las labores de la Comisión, regulado en el Reglamento (UE) n. ${ }^{o}$ 182/2011, así como la inaplicación de estas medidas una vez que se hayan adoptado las correcciones oportunas por el Estado implicado. 


\subsection{Actos del Consejo}

A. Reglamento n. ${ }^{\circ}$ 1243/2012 del Consejo, de 19 de diciembre de 2012, que modifica el Reglamento (CE) n. ${ }^{\circ} 1342 / 2008$ por el que se establece un plan a largo plazo para las poblaciones de bacalao y las pesquerías que las explotan

El Reglamento establece un plan a largo plazo para las poblaciones de bacalao del Kattegat, del mar del Norte, Skagerrak y Mancha oriental, del oeste de Escocia y del mar de Irlanda, así como para las pesquerías que las explotan (en lo sucesivo, Plan del Bacalao). El objetivo del Plan del Bacalao es garantizar la explotación sostenible de esas poblaciones de bacalao sobre la base del rendimiento máximo sostenible. Este objetivo debe alcanzarse manteniendo al mismo tiempo un nivel específico de mortalidad por pesca del bacalao en grupos de edad apropiados.

La evaluación científica de los resultados del Plan del Bacalao realizada por el Comité Científico, Técnico y Económico de Pesca (CCTEP) ha puesto de manifiesto que la concepción y el funcionamiento del Plan plantean una serie de problemas que el citado Reglamento busca abordar. Sin poner en tela de juicio los objetivos de dicho Plan, el CCTEP concluyó que es improbable que tales objetivos sean alcanzados dentro de un marco temporal que sea compatible con las conclusiones de la Cumbre Mundial sobre el Desarrollo Sostenible celebrada en Johannesburgo en 2002, a no ser que se corrijan los defectos de concepción del Plan referidos, entre otras cosas, a la aplicación de sus artículos 9 y 12. En este sentido, es esencial modificar urgentemente tales preceptos del Plan del Bacalao para que puedan aplicarse las nuevas normas a efectos de la fijación de las posibilidades de pesca para 2013. Esta modificación es la que lleva a cabo el Reglamento que nos ocupa.

Otros actos jurídicos a destacar, todos ellos en el marco de la protección de especies de pesca o de los ecosistemas marinos, son los siguientes:

- Reglamento n. ${ }^{\circ}$ 1088/2012 del Consejo, de 20 de noviembre de 2012, por el que se establecen, para 2013, las posibilidades de pesca aplicables en el mar Báltico a determinadas poblaciones y grupos de poblaciones de peces.

- Decisión del Consejo, de 16 de julio de 2012, relativa a la firma, en nombre de la Unión Europea, y a la aplicación provisional del Protocolo por el que se fijan las posibilidades de pesca y la contrapartida financiera previstas en el Acuerdo de 
colaboración en materia de pesca entre la Comunidad Europea, por una parte, y el Gobierno de Dinamarca y el Gobierno Autónomo de Groenlandia, por otra.

— Decisión del Consejo, de 17 de diciembre de 2012, sobre la adhesión de la Unión Europea al Protocolo para la protección del mar Mediterráneo contra la contaminación resultante de la exploración y explotación de la plataforma continental, del fondo del mar y de su subsuelo (2013/5/UE).

\subsection{Actos del Parlamento}

A. Resolución del Parlamento Europeo, de 29 de septiembre de 2011, sobre la elaboración de una posición común de la UE ante la Conferencia de las Naciones Unidas sobre el Desarrollo Sostenible (Río+20) (2013/C 56 E/14)

En relación con la Conferencia de las Naciones Unidas sobre el Desarrollo Sostenible (Río+20), que se celebró en Río de Janeiro en junio de 2012 y que se centró en dos temas cruciales como la "economía ecológica en el contexto del desarrollo sostenible y la erradicación de la pobreza" y el "marco institucional para el desarrollo sostenible", el Parlamento aplaude en la presente Resolución la decisión de la Asamblea General de las Naciones Unidas, plasmada en la Resolución 64/236, de convocar una conferencia de las Naciones Unidas sobre el desarrollo sostenible al nivel más alto posible (jefes de Estado y/o de Gobierno).

La Resolución considera que esa conferencia representa una oportunidad única para que los líderes mundiales elaboren la agenda de sostenibilidad para los próximos diez años, al tiempo que reafirma la necesidad de la solidaridad mundial. El Parlamento destaca que debe ponerse el desarrollo sostenible en primer término para todos los procesos y políticas de la UE, a fin de que la Unión sea coherente tanto internamente como en sus aspiraciones internacionales, haciendo hincapié en que hay una necesidad crucial de inyectar mayor urgencia y dinamismo a la puesta en marcha de la gobernanza internacional de las políticas de desarrollo sostenible, que están progresando demasiado lentamente.

Se insiste en que la "economía ecológica" —uno de los dos temas principales de la Cumbre - debe entenderse como el funcionamiento económico en su conjunto dentro de los límites de la sostenibilidad en cuanto a biodiversidad, mantenimiento de los servicios ecosistémicos, protección del clima y uso de recursos naturales. Se destaca, 
igualmente, que debe prestarse más atención al capital humano, medioambiental y natural, y que el desarrollo sostenible es más que una simple "economía ecológica". Igualmente, se resalta la necesidad de mejorar la gobernanza y reforzar la protección del medio marino, la biodiversidad marina y los océanos. A tal efecto, se considera que los mares y océanos deben convertirse, junto con la protección del clima y de la biodiversidad, en uno de los pilares fundamentales del Marco de Río.

B. Resolución del Parlamento Europeo, de 14 de septiembre de 2011, sobre un enfoque global con respecto a las emisiones antropogénicas de gases distintos al $\mathrm{CO}_{2}$ que afectan al clima (2013/C $51 \mathrm{E} / 14)$

El Parlamento aboga, mediante esta resolución, por una política europea global en el ámbito del clima que se beneficie de la toma en consideración de todas las fuentes del calentamiento global y de todas las alternativas para mitigarlo; asimismo, opina que, además de tener en cuenta la reducción de las emisiones de $\mathrm{CO}_{2}$, dicha política debería hacer hincapié en las estrategias que ofrezcan una respuesta lo más rápida posible en el ámbito del clima.

Señala la Resolución que existen estrategias reguladoras de acción rápida para reducir gradualmente la producción y el consumo de HFC y para disminuir las emisiones de negro de carbón y de los gases que llevan a la formación del ozono troposférico, estrategias que podrían iniciarse en un plazo de dos a tres años y aplicarse de manera sustancial a lo largo de los próximos cinco a diez años, lo que brindaría una respuesta adecuada en materia climática, en algunos decenios o incluso antes, en particular para algunos HFC con un precio público muy bajo ( 5 a 10 céntimos por tonelada).

La Resolución insta a la Comisión para que presente una revisión de los reglamentos sobre gases fluorados y propuestas dirigidas a reducir de forma rápida y gradual la producción y el consumo de HFC, a acelerar la eliminación de los hidroclorofluorocarburos (HCFC) en diferentes productos y aplicaciones y a recuperar y destruir de los productos y equipos desechados los gases de efecto invernadero que agotan el ozono estratosférico.

Para concluir, acoge con satisfacción el compromiso de la Unión Europea de apoyar, en la COP 17 que tendrá lugar en Durban, las medidas relativas a los HFC en el marco del 
Protocolo de Montreal por cuanto son un ejemplo excelente de un enfoque no basado en el mercado para reducir las emisiones de gases de efecto invernadero.

C. Resolución del Parlamento Europeo, de 11 de mayo de 2011, sobre el Libro Verde de la Comisión sobre protección de los bosques e información forestal en la UE: preparación de los bosques al cambio climático (2010/2106(INI)) (2012/C 377 E/05)

El Parlamento acoge favorablemente el Libro Verde de la Comisión sobre protección de los bosques e información forestal en la UE. Considera que debe reforzarse la estrategia forestal de la Unión con vistas a mejorar la gestión y conservación sostenibles de los bosques, de conformidad con los principios de subsidiariedad y proporcionalidad. Por otro lado, sostiene que, según el artículo 5 del Tratado de la Unión Europea, la UE puede intervenir en los ámbitos en los que se demuestre que los objetivos de la acción propuesta no puedan ser alcanzados de manera suficiente por los Estados miembros.

Recuerda que los bosques constituyen biosferas cuya resistencia depende de la diversidad biológica no solo de los árboles, sino también de todos los organismos forestales, en particular los animales silvestres que viven en los bosques. Asimismo, se afirma que los bosques constituyen el principal sumidero de carbono, desempeñando un papel primordial en la lucha contra el cambio climático; es, por lo tanto, de crucial importancia, afirma la Resolución, que la Unión Europea refuerce su estrategia para luchar contra los fenómenos que deterioran la superficie forestal, como los incendios o la contaminación atmosférica.

Expresa su convencimiento de que la sostenibilidad ecológica es una condición previa indispensable para la continuidad de las funciones económicas y sociales de los bosques de la UE. Se hace hincapié, igualmente, en que deben actualizarse la Estrategia Forestal de la UE y el Plan de Acción de la UE para los Bosques con objeto de incluir la dimensión del cambio climático y temas más amplios de protección de los bosques. Se recuerda, asimismo, que antes de proceder a tal revisión debe celebrarse un amplio debate sobre política forestal con los Estados miembros y todas las partes interesadas en la aplicación de las medidas propuestas.

Finalmente, el Parlamento pide a la Comisión y a los Estados miembros que redoblen los esfuerzos dirigidos a alcanzar los objetivos del Plan de Acción de la UE para los 
Bosques en el ámbito del medio ambiente y la calidad de vida, al tiempo que recuerda el retraso en la aplicación de dicho Plan.

\subsection{Actos de la Comisión}

A. Reglamento n. ${ }^{o}$ 1158/2012 de la Comisión, de 27 de noviembre de 2012, que modifica el Reglamento (CE) n. ${ }^{\circ}$ 338/97 del Consejo, relativo a la protección de especies de la fauna y flora silvestres mediante el control de su comercio

Las modificaciones realizadas en el apéndice III de la Convención sobre el Comercio Internacional de Especies Amenazadas de Fauna y Flora Silvestres (CITES) hacen necesario realizar cambios en el anexo C del anexo del Reglamento (CE) 338/97 para añadir determinadas especies.

B. Directiva 2012/40/UE de la Comisión, de 26 de noviembre de 2012, por la que se corrige el anexo I de la Directiva 98/8/CE del Parlamento Europeo y del Consejo, relativa a la comercialización de biocidas

La Directiva 2009/91/CE de la Comisión, de 31 de julio de 2009, por la que se modificó la Directiva 98/8/CE del Parlamento Europeo y del Consejo, en virtud de la cual se incluyó el tetraborato de disodio como sustancia activa en el anexo I de la Directiva, define el tetraborato de disodio mediante tres números CAS correspondientes a tres formas diferentes de la sustancia. Los números CAS se basan en un informe presentado a la Comisión por los Países Bajos el 7 de julio de 2006 y aprobado por el Comité Permanente de Biocidas el 20 de febrero de 2009.

Los Países Bajos informaron a la Comisión de que el número CAS de la forma pentahidratada que figuraba en el informe original era incorrecto y, por ello, presentaron a la Comisión un informe revisado según el cual el número CAS correcto de esa forma era 12179-04-3. El informe revisado fue aprobado por el Comité Permanente de Biocidas el 25 de mayo de 2012. 
C. Dictamen de la Comisión, de 18 de diciembre de 2012, sobre el plan de eliminación de los residuos radiactivos producto del desmantelamiento de la central nuclear de KLW, situada en Lingen, Baja Sajonia, Alemania

La Comisión considera que no es probable que la aplicación del plan de evacuación de los residuos radiactivos, del tipo que sea, producto del desmantelamiento de la central nuclear de KLW, situada en Lingen, Baja Sajonia (Alemania), tanto en caso de funcionamiento normal como en caso de accidente del tipo y magnitud considerados en los datos generales, cause una contaminación radiactiva del agua, el suelo o el espacio aéreo de otro Estado miembro que sea significativa desde el punto de vista sanitario. Sin embargo, en situaciones de accidente más grave, la dosis que afectaría a la población que vive en las zonas fronterizas del Estado miembro más próximo podría alcanzar niveles que llevaran a las autoridades competentes a considerar la adopción de medidas de control. En este contexto, cabe señalar que Alemania y los Estados vecinos han ratificado acuerdos bilaterales sobre pronta notificación y asistencia mutua en caso de emergencia radiológica.

D. Decisión de Ejecución de la Comisión, de 19 de febrero de 2013, relativa a las limitaciones a las autorizaciones de biocidas que contengan difenácum notificadas por Alemania de conformidad con la Directiva 98/8/CE del Parlamento Europeo y del Consejo (2013/96/UE)

Mediante esta decisión se permite a Alemania limitar las autorizaciones concedidas sobre los biocidas para uso por profesionales formados o titulares de una licencia, sin excluir la protección alimentaria de los usos previstos autorizados de los biocidas.

E. Decisión de la Comisión, de 13 de diciembre de 2012, relativa a las disposiciones nacionales notificadas por Austria sobre determinados gases industriales de efecto invernadero $(2012 / 784 / \mathrm{UE})$

La Comisión aprueba la solicitud de Austria, presentada el 27 de junio de 2012, de mantener, en relación con la comercialización de productos y aparatos que contengan gases fluorados o que dependan de estos, una legislación nacional más estricta que el Reglamento n. ${ }^{\circ} 842 / 2006$, con el fin de conseguir sus compromisos marcados en el Protocolo de Kioto. En este sentido, la Comisión sostiene que, a pesar de que la medida 
nacional austriaca tiene implicaciones para la libre circulación de mercancías en la Unión, las disposiciones son de carácter general y se aplican de igual modo a los productos nacionales y a los importados. Por lo tanto, se entiende que no parece que sean discriminatorias entre los agentes económicos de la Unión. Asimismo, dado el objetivo medioambiental que persigue la Orden nacional, no constituye un obstáculo desproporcionado en relación con los objetivos que pretende alcanzar.

F. Decisión de la Comisión, de 14 de noviembre de 2012, por la que se cursa una notificación a los terceros países que la Comisión estima susceptibles de ser considerados terceros países no cooperantes conforme al Reglamento (CE) n. ${ }^{\circ}$ 1005/2008 del Consejo por el que se establece un sistema comunitario para prevenir, desalentar y eliminar la pesca ilegal, no declarada y no reglamentada (2012/C 354/01)

Conforme al artículo 32 del Reglamento INDNR, la Comisión debe cursar una notificación a los terceros países susceptibles de ser considerados países no cooperantes. Dicha notificación tiene carácter preliminar. La notificación a esos países de la posibilidad de ser considerados terceros países no cooperantes debe estar basada en los criterios establecidos en el artículo 31 del Reglamento INDNR. En particular, la Comisión debe incluir en la notificación información relativa a los principales hechos y consideraciones que sustentan tal identificación y a la posibilidad para los países en cuestión de presentar alegaciones y pruebas en contrario o, en su caso, un plan de actuación para mejorar la situación y las medidas adoptadas para corregirla. La Comisión debe conceder a los terceros países afectados el tiempo necesario para responder a la notificación y un plazo razonable para poner remedio a la situación. La identificación de terceros países no cooperantes se basará en un análisis de toda la información obtenida conforme al artículo 31.2 del Reglamento INDNR. De conformidad con el artículo 33 del citado Reglamento, el Consejo podrá elaborar una lista de países no cooperantes.

En este sentido, en la presente Decisión se notifica a la República de Belice, el Reino de Camboya, la República de Fiyi, la República de Guinea, la República de Panamá, la República Socialista Democrática de Sri Lanka, la República de Togo y la República de Vanuatu la posibilidad de ser considerados por la Comisión terceros países no cooperantes en la lucha contra la pesca ilegal, no declarada y no reglamentada. 
G. Decisión de Ejecución de la Comisión, de 23 de octubre de 2012, por la que se concede la exención solicitada por el Reino de Dinamarca de conformidad con la Directiva 91/676/CEE del Consejo, relativa a la protección de las aguas contra la contaminación producida por nitratos utilizados en la agricultura (2012/659/UE)

A través de la presente Decisión se concede la exención solicitada por el Reino de Dinamarca, mediante carta de 20 de junio de 2012, para permitir la aplicación de una cantidad de estiércol superior a la prevista en el anexo III, punto 2, párrafo segundo, primera frase, de la Directiva 91/676/CEE, así como en la letra a) de ese mismo párrafo. La presente exención se aplicará, con carácter individual y en las condiciones establecidas en los artículos 4 a 6 de la Decisión, a las explotaciones de ganado vacuno en las que la rotación de cultivos comprenda más de un $70 \%$ de cultivos con elevada captación de nitrógeno y ciclos de crecimiento largos. Los ganaderos presentarán cada año una solicitud de exención a las autoridades competentes.

En la Decisión se fijan igualmente determinados límites a la aplicación de estiércol y otros abonos, así como en la gestión de las tierras. Asimismo, se establece un seguimiento de las exenciones y un control administrativo que velará por la correcta aplicación de estas exenciones.

H. Decisión de Ejecución de la Comisión, de 23 de noviembre de 2012, sobre el reconocimiento del régimen "Roundtable on Sustainable Palm Oil RED" para demostrar el cumplimiento de los criterios de sostenibilidad con las directivas 98/70/CE y 2009/28/CE del Parlamento Europeo y del Consejo (2012/722/UE)

Se establece que la solicitud sobre la aplicación de un régimen voluntario especial denominado "Roundtable on Sustainable Palm Oil RED" cumple los criterios de sostenibilidad establecidos en las directivas 98/70/CE y 2009/28/CE del Parlamento Europeo y del Consejo. La Decisión avala este régimen voluntario por un plazo de cinco años, salvo que se produjesen modificaciones en este, en cuyo caso habrá que notificarlas a la Comisión, que evaluará si el régimen modificado sigue cumpliendo adecuadamente los criterios de sostenibilidad. 
I. Decisión de Ejecución de la Comisión, de 14 de noviembre de 2012, por la que se establece un formato común para la presentación de la información prevista en la Directiva 2010/63/UE del Parlamento Europeo y del Consejo, relativa a la protección de los animales utilizados para fines científicos (2012/707/UE)

En la Decisión que aquí nos ocupa se adjuntan varios anexos que buscan unificar el formato para presentar la comunicación, la información estadística y la información sobre las exenciones previstas en la Directiva 2010/63/UE.

Otras decisiones dictadas en el período analizado son las citadas a continuación:

— Decisión de la Comisión, de 14 de noviembre de 2012, por la que se establecen los criterios ecológicos para la concesión de la etiqueta ecológica de la UE a los detergentes para ropa de uso industrial e institucional (2012/721/UE).

- Decisión de Ejecución de la Comisión, de 4 de diciembre de 2012, por la que se modifica el anexo I de la Decisión 2009/177/CE en lo que respecta a los programas de vigilancia para Finlandia y el Reino Unido y a la clasificación de "libre de la enfermedad" de Finlandia y el Reino Unido en relación con determinadas enfermedades de los animales acuáticos (2012/753/UE).

— Decisión de Ejecución de la Comisión, de 11 de diciembre de 2012, por la que se determinan los límites cuantitativos y se asignan cuotas de sustancias reguladas de conformidad con el Reglamento n. ${ }^{\circ}$ 1005/2009 del Parlamento Europeo y del Consejo, sobre las sustancias que agotan la capa de ozono, para el período comprendido entre el 1 de enero y el 31 de diciembre de 2013 (2012/782/UE).

- Decisión de Ejecución de la Comisión, de 12 de diciembre de 2012, por la que se establecen el tipo, el formato y la frecuencia de la información que deben comunicar los Estados miembros sobre la aplicación de la Directiva 2010/75/UE del Parlamento Europeo y del Consejo, sobre las emisiones industriales (2012/795/UE).

\subsection{Otros}

A. Anuncio de la Comisión Europea a las empresas que tengan la intención de importar a la Unión, o exportar desde esta, sustancias reguladas que agotan la capa de ozono en 2014 y a las empresas que tengan la intención de producir o importar esas sustancias para usos esenciales de laboratorio y análisis en 2014 (2013/C 25/06) 
Este anuncio se dirige a todas las empresas a las que se refiere el Reglamento $\mathrm{n}^{\circ}$ 1005/2009 sobre las sustancias que agotan la capa de ozono y se refiere, asimismo, al grupo de sustancias específicas previstas en su anexo I. En este sentido, toda importación o exportación de estas sustancias exige una licencia de la Comisión, y su producción requiere una autorización previa.

Asimismo, algunas actividades quedarán sujetas a limitaciones cuantitativas en virtud de las cuotas asignadas previamente por la Comisión. Todas aquellas actividades que no se vean afectadas por estas limitaciones deberán adecuarse al procedimiento previsto, por el cual se deberá informar a la Comisión de su propósito registrándose en el sistema ODS Licensing System para solicitar su cuota. Entre las novedades destaca el cambio de programa para solicitar las cuotas y expedir las licencias, el cual será el ODS Licensing System.

B. Dictamen del Comité de las Regiones: "La innovación al servicio del crecimiento sostenible: una bioeconomía para Europa" (2013/C 17/09)

Tomando como texto de referencia la Comunicación de la Comisión al Parlamento Europeo, al Consejo, al Comité Económico y Social Europeo y al Comité de las Regiones: "La innovación al servicio del crecimiento sostenible: una bioeconomía para Europa” COM (2012) 60 final, el Comité de las Regiones constata que:

- Las ciencias de la vida y la biotecnología contribuyen de manera considerable a cumplir los objetivos fundamentales de las políticas de la UE en ámbitos como la salud, el desarrollo sostenible y económico y la creación de empleo.

- La transición a la bioeconomía es un requisito previo para que Europa se convierta en un líder mundial en el ámbito de la bioeconomía, especialmente en lo relativo a la innovación y la competitividad.

— En Horizonte 2020 la bioeconomía debería considerarse un "reto social" y ser objeto de un presupuesto propuesto por la Comisión de 4.500 millones de euros.

- Pasarán todavía al menos veinticinco años antes de que la bioeconomía pueda competir con la economía basada en los combustibles fósiles, y que ello requiere una inversión a largo plazo (en I+D), estrategias (para después de 2020) y cooperación entre 
todas las partes interesadas a lo largo de la cadena de valor con el objetivo de lograr la transferencia de conocimiento cooperativo.

— La bioeconomía proporcionará nuevas oportunidades de negocio e innovación para la cadena de valor europea, incluido el sector agrícola.

- Los instrumentos de ordenación territorial son importantes para preservar las superficies utilizadas con fines agrarios o forestales.

- El plan de acción propuesto por la Comisión no contempla ninguna medida para explotar más eficazmente los recursos naturales.

C. Dictamen del Comité Económico y Social Europeo sobre la "Comunicación de la Comisión al Parlamento Europeo, al Consejo, al Comité Económico y Social Europeo y al Comité de las Regiones - Innovación para un futuro sostenible. Plan de Acción sobre Ecoinnovación (Eco-AP)” [COM (2011) 899 final], (2012/ C 351/14)

El Comité respalda el plan de acción mencionado en el Dictamen que aquí nos ocupa. Se considera que la innovación ecológica es un ejercicio de gran importancia, si no el más importante, para mantener la competitividad a largo plazo y alcanzar los objetivos de la sostenibilidad, pero también para mostrar a las regiones menos desarrolladas la manera de avanzar hacia un desarrollo económico y una prosperidad que no dañen el medio ambiente.

Una cuestión relevante es la del significado exacto del concepto de ecoinnovación. Lo que una persona o una cultura pueden considerar "innovador" o "avanzado" puede suscitar más bien rechazo en otras personas o culturas. Esta situación se ilustra claramente con los ejemplos de la genómica o el uso de la energía nuclear. De ello cabe deducir que no existe una definición de validez universal de ecoinnovación.

El CESE recomienda además a la Comisión que reflexione sobre la posibilidad de definir, en el marco del plan de acción, áreas prioritarias que deban promoverse con carácter preferente. Podría tratarse de cualquier sector de la política medioambiental en el que a) Europa haya hecho solo progresos muy escasos desde hace muchos años, b) sea evidente que existen problemas graves para lograr cumplir los objetivos ambientales y c) la tecnología siga resultando igual de cara. 
A tal efecto, la Comisión debería considerar la posibilidad de imponer a todos los proyectos de financiación la obligación de realizar un breve estudio adicional en el que se indiquen cuáles son los obstáculos reales o potenciales. El tratamiento de los temas no tendría por qué ser de un nivel científico muy elevado, sino que se trataría simplemente de aportar indicaciones que muestren a los decisores políticos las áreas en las que hay necesidades suplementarias de aplicación. Al mismo tiempo es preciso que la Comisión revise a intervalos regulares todas sus directivas y reglamentos anteriores, pero también los criterios de subvencionabilidad de los Fondos Estructurales y de la Política Agrícola Común, con objeto de verificar si es preciso adaptarlos a las innovaciones tecnológicas medioambientales más recientes.

Para terminar, el CESE señala que en este plan de acción - como en muchos otros documentos - se afirma que hay que suprimir las subvenciones con efectos perjudiciales para el medio ambiente. Por esta razón, el CESE considera aún más irritante que la Comisión, pese a las numerosas admoniciones del Comité, siga sin presentar la correspondiente lista de subvenciones con efectos perjudiciales para el medio ambiente tras más de cinco años de promesas.

D. Dictamen del Comité Económico y Social Europeo sobre la "Propuesta de Reglamento del Parlamento Europeo y del Consejo, por el que se modifica el Reglamento (CE) n. ${ }^{o}$ 850/98 del Consejo para la conservación de los recursos pesqueros a través de medidas técnicas de protección de los juveniles de organismos marinos y se deroga el Reglamento (CE) n. ${ }^{\circ} 1288 / 2009$ del Consejo" [COM(2012) 298 final 2012/0158 (COD)] (2012/C 351/18)

El Consejo, con fecha 5 de julio de 2012, y el Parlamento Europeo, con fecha 10 de julio de 2012, de conformidad con el artículo 43 del Tratado de Funcionamiento de la Unión Europea, consultaron al Comité Económico y Social Europeo sobre la Propuesta de Reglamento del Parlamento Europeo y del Consejo por el que se modifica el Reglamento (CE) n. ${ }^{o}$ 850/98 del Consejo para la conservación de los recursos pesqueros a través de medidas técnicas de protección de los juveniles de organismos marinos y se deroga el Reglamento (CE) n. ${ }^{\circ}$ 1288/2009 del Consejo.

Dado que las medidas técnicas temporales que figuran en el Reglamento del Consejo (CE) n. ${ }^{\circ}$ 1288/2009 dejarán de ser aplicables el 31 de diciembre de 2012, el Comité 
Económico y Social Europeo está de acuerdo con la propuesta de la Comisión para garantizar la seguridad jurídica mientras se elabora un nuevo reglamento marco de medidas técnicas dentro de la reforma de la Política Pesquera Común.

Estas medidas técnicas son importantes para asegurar una pesca sostenible, por lo que debe garantizarse su continuidad. Interrumpir (incluso temporalmente) dichas medidas tendría consecuencias negativas en la conservación de las poblaciones a las que se aplican y en el ecosistema de hábitats vulnerables de aguas profundas, incluyendo una serie de lugares de la red Natura 2000. Su interrupción entrañaría asimismo la supresión de ciertas excepciones, justificadas y aceptadas, a las disposiciones del Reglamento (CE) n. ${ }^{\circ} 850 / 98$.

El Comité sugiere prorrogar la aplicación de las medidas técnicas transitorias del Reglamento (CE) n. ${ }^{\circ}$ 1288/2009 durante un período adicional de 18 meses, en lugar de incorporarlas al Reglamento (CE) n. ${ }^{\circ} 850 / 98$.

E. Dictamen del Comité Económico y Social Europeo sobre la "Propuesta de Reglamento del Parlamento Europeo y del Consejo, que modifica el Reglamento (CE) n. ${ }^{\circ}$ 1005/2008 del Consejo, por el que se establece un sistema comunitario para prevenir, desalentar y eliminar la pesca ilegal, no declarada y no reglamentada" [COM(2012) 332 final — 2012/162 (COD)], (2012/C 351/21)

Considerando que el contenido de la propuesta era satisfactorio, el Comité decidió, por 141 votos a favor, ningún voto en contra y 7 abstenciones, emitir un dictamen favorable sobre el texto propuesto.

F. Dictamen del Comité de las Regiones - Fondo Europeo Marítimo y de Pesca (FEMP) (2012/C 391/10)

Este dictamen aprueba la creación del nuevo Fondo Europeo Marítimo y de Pesca (FEMP) con vistas a ejecutar la Política Pesquera Común (PPC), y considera importante preservar el presupuesto indispensable para acompañar la evolución impuesta por la PPC; asimismo, se congratula por la simplificación que supone agrupar en el nuevo FEMP la mayoría de los instrumentos financieros de la PPC y de la Política Marítima Integrada (PMI), dispersos anteriormente entre distintos fondos. 
Igualmente, se solicita que los objetivos del FEMP se enfoquen hacia la pesca y no se centren prioritariamente, como se ha afirmado varias veces, en sustituirla por otras actividades. Además, considera importante reforzar el atractivo del oficio de pescador y muestra su inquietud por la reducción del presupuesto asignado a la recopilación de datos, ya que se necesitarán medios adicionales. Disponer de información exhaustiva y elaborada a efectos de gestión debería ser, por una parte, un requisito previo de la PPC $\mathrm{y}$, por otra, una prioridad presupuestaria del FEMP.

El CESE condena la supresión de cualquier medida de ajuste de la flota, pese a que para ajustarse a los nuevos objetivos de la PPC, en particular la consecución gradual del rendimiento máximo sostenible (RMS), será necesario desmantelar buques o financiar paralizaciones temporales de pesca.

Considera que, para aplicar la reducción progresiva de los descartes, será preciso adaptar y modernizar los barcos, así como realizar las inversiones portuarias adecuadas; a su vez, manifiesta su extrañeza por la falta de financiación para elaborar los planes plurianuales previstos.

Para finalizar, solicita que se ayude decididamente a la innovación tecnológica y a las inversiones que refuerzan la selectividad de las artes de pesca, considerando que la reducción progresiva de las ayudas al almacenamiento no es pertinente.

G. Dictamen del Comité Económico y Social Europeo sobre la "Propuesta de Reglamento del Parlamento Europeo y del Consejo que modifica el Reglamento (CE)

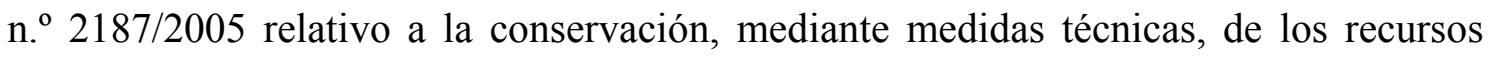
pesqueros en aguas del mar Báltico, los Belts y el Sund" [COM (2012) 591 final 2012/0285 (COD)] (2013/C 44/28)

Considerando que el contenido de la propuesta es satisfactorio, el Comité decidió, por 129 votos a favor y 1 abstención, emitir un dictamen favorable sobre el texto propuesto. 
H. Dictamen del Comité Económico y Social Europeo sobre la "Comunicación de la Comisión al Parlamento Europeo, al Consejo, al Comité Económico y Social Europeo y al Comité de las Regiones relativa a la Cooperación de Innovación Europea sobre el Agua" [COM (2012) 216 final] (2013/C 44/26)

El CESE acoge favorablemente la Comunicación de la Comisión Europea relativa a la cooperación de innovación europea (CIE) sobre el agua, pero propone aclaraciones y mejoras con el fin de contribuir a eliminar los obstáculos que impiden un buen desarrollo de la innovación en el sector del agua.

La innovación en este ámbito en Europa debería basarse en un enfoque integral que tenga en cuenta la totalidad del ciclo del agua y se centre prioritariamente en alcanzar en toda Europa el "buen estado de las aguas superficiales y subterráneas", tal como se define en la Directiva Marco del Agua, y en reforzar la protección de este recurso aplicando el principio de "quien contamina, paga", suficientemente disuasorio para evitar la contaminación y la impunidad.

El Plan Estratégico de Aplicación de las prioridades de la CIE debe abordar el hecho de que en Europa más de un millón de personas no tienen acceso a agua segura, limpia y asequible, y que varios millones de ciudadanos carecen de cualquier tipo de saneamiento. Las necesidades de estas personas son una prioridad desde una perspectiva de inclusión y lucha contra la pobreza.

Los proveedores de servicios públicos de agua, los usuarios y los consumidores deben contar con la posibilidad de hacer oír su voz con fuerza en el proceso decisorio en torno a la CIE sobre el agua. Asimismo, es necesario que la CIE sobre el agua se traduzca en una mejora de la coordinación de todos los operadores, que los beneficios de la innovación alcancen al nivel local y que se facilite la participación de las organizaciones representativas de la sociedad civil en las nuevas redes y grupos que se creen.

Para el CESE es necesario dar acceso de manera transparente a los resultados de la investigación financiada por el VII Programa Marco de Investigación y Desarrollo a fin de crear asociaciones para la innovación sobre el agua, habida cuenta del carácter específico y vital del agua para la población. Por otro lado, desaconseja tratar las innovaciones en este ámbito sensible únicamente desde el punto de vista de la protección comercial, y recomienda facilitar por igual el acceso tanto a las instancias, 
los organismos y los entes territoriales locales como a las empresas de la economía social.

En este sentido, el CESE pide a la Comisión que redoble sus esfuerzos para garantizar la transparencia y la coordinación de determinadas iniciativas importantes en curso relacionadas con la vasta problemática del agua. Por ejemplo, es necesario que la Comisión aporte precisiones sobre las sinergias y el funcionamiento conjunto de las CIE recientes sobre el agua, la agricultura y las materias primas.

No habrá una verdadera política de investigación e innovación sobre el agua sin transparencia ni tampoco sin una política de empleo integradora que garantice desde el principio suficiente personal, formación, reconocimiento de las cualificaciones y tecnologías que permitan mejorar la salud y la seguridad tanto en los procesos de purificación y tratamiento del agua como en los de saneamiento, y que garanticen al máximo el conjunto de las misiones en toda su diversidad y a todos los niveles.

Para terminar, el CESE subraya el papel de las redes de organizaciones de la sociedad civil (OSC), que debería reconocerse y valorarse adecuadamente y constituir, asimismo, un ámbito de investigación sobre el potencial de innovación que representan estas OSC por su experiencia y su capital de conocimientos.

I. Dictamen del Comité Económico y Social Europeo sobre la "Comunicación de la Comisión al Consejo y al Parlamento Europeo sobre las evaluaciones completas del riesgo y de la seguridad ('pruebas de resistencia') de las centrales nucleares de la Unión Europea y actividades relacionadas" [COM (2012) 571 final]. (2013/C 44/25)

Aunque la gestión de los riesgos depende esencialmente de la solidez de las centrales nucleares, el CESE estima que hay que considerar todos los riesgos, incluidos los externos, que suponen las centrales para los ciudadanos, el medio ambiente y la economía. El CESE considera indispensable prever, para cada emplazamiento, la gestión de accidentes, incluida la formación del personal, la información y la consulta a los habitantes de los alrededores. Se debe permitir que estos últimos participen en la elaboración de las consignas y aprovechar su conocimiento del terreno. Conviene prever, incluso, una gestión posterior al accidente, lo que corresponde a un enfoque a largo plazo. 
Asimismo, respalda la iniciativa de la Comisión de emprender una revisión ambiciosa de la Directiva sobre seguridad nuclear y le pide que se comprometa a no circunscribirse únicamente a los aspectos técnicos, sino también a considerar los aspectos humanos relativos tanto a los trabajadores como a los ciudadanos (salud, estrés, aspectos psicológicos, etc.).

El mismo dictamen constata que no todos los Estados miembros disponen de una autoridad reguladora independiente, dotada de potestad reglamentaria, y que entre los Estados miembros no hay un enfoque común en materia de reglamentación sobre seguridad nuclear, razón por la cual el CESE recomienda realizar una armonización mediante la Directiva.

La información al público y la participación de los ciudadanos debería basarse en el Convenio de Aarhus aplicado al ámbito nuclear, que regula la información, la participación o concertación y el acceso a la justicia. Este convenio ha sido firmado por la UE y los Estados miembros.

El CESE considera que, a raíz de las pruebas de resistencia y de las recomendaciones realizadas por la Comisión, la UE debe establecer mecanismos de seguimiento y verificación que incluyan la presentación por parte de los Estados miembros de informes periódicos a escala europea, e insiste en la importancia de la cooperación estrecha y del intercambio de información entre operadores, vendedores, autoridades reguladoras e instituciones europeas preconizados en la Comunicación, colaboración que debe extenderse a los ciudadanos, así como al personal y a sus representantes, en particular en las zonas fronterizas, donde es preciso armonizar los procedimientos.

El Dictamen respalda la iniciativa de la Comisión de proponer un acto legislativo sobre los seguros y la responsabilidad en el ámbito nuclear, los cuales en la actualidad no cubren realmente los riesgos. En efecto, el CESE considera que debe abarcar, en particular, los aspectos sociales, medioambientales y económicos mediante fondos que deberán alimentar los productores europeos de electricidad nuclear en Europa. Por otro lado, se corre el riesgo de que las posibles víctimas no sean protegidas, ni tampoco indemnizadas, de manera suficiente.

Para concluir, recomienda a la Comisión instaurar una profilaxis armonizada de las lesiones de tiroides causadas por la ingestión de yodo estable en caso de accidente grave que sea válida para todo el territorio de la UE, así como ampliar, según las lecciones 
extraídas de Fukushima, a 20 o $30 \mathrm{~km}$ las zonas de evacuación en los alrededores de los emplazamientos europeos densamente poblados.

J. Dictamen del Comité Económico y Social Europeo sobre la "Propuesta de Reglamento del Parlamento Europeo y del Consejo que modifica el Reglamento (CE)

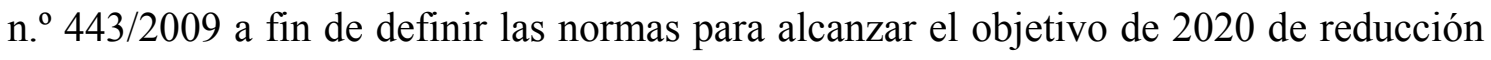
de las emisiones de $\mathrm{CO}_{2}$ de los turismos nuevos [COM (2012) 393 final - 2012/0190 (COD)] en virtud de la Propuesta de Reglamento del Parlamento Europeo y del Consejo por el que se modifica el Reglamento (UE) n. ${ }^{\circ}$ 510/2011 a fin de establecer las normas para alcanzar el objetivo de 2020 de reducción de las emisiones de $\mathrm{CO}_{2}$ de los vehículos comerciales ligeros nuevos" [COM (2012) 394 final - 2012/0191 (COD)], (2013/C 44/19)

En sus dictámenes, el CESE siempre ha apoyado todas las iniciativas de la Comisión para reducir las emisiones de $\mathrm{CO}_{2}$ con el objetivo de conseguir una Europa libre de carbono antes de 2050, para lo cual el sector del transporte debería reducir sus propias emisiones en un $60 \%$.

El CESE recuerda que en febrero de 2011 el Consejo Europeo confirmó el objetivo de la UE de reducir, antes de 2050, las emisiones de gases de efecto invernadero en un 8095\% respecto a los niveles de 1990. Según el Grupo Intergubernamental de Expertos sobre el Cambio Climático, los países desarrollados deben alcanzar colectivamente los objetivos de reducción, teniendo en cuenta los esfuerzos necesarios por parte de los países en desarrollo. Esto debería permitir la reducción de las emisiones en un 50\% a nivel mundial antes de 2050, siguiendo un objetivo de reducción para el período posterior a Kioto en un marco jurídico global que deberá adoptarse antes de 2015 y aplicarse a partir de 2020 .

El CESE comparte la opinión de establecer objetivos cada vez más rigurosos para la lucha contra el cambio climático, principalmente para el sector del transporte en carretera, que supone el $24 \%$ de las emisiones totales de la UE de anhídrido carbónico $\left(\mathrm{CO}_{2}\right)$, principal gas de efecto invernadero (GEI) cuyas emisiones han aumentado casi un $23 \%$ entre 1990 y 2010 . Esta tendencia no es sostenible a la luz de la política climática de la Unión Europea. Para ello, subraya la necesidad de actuar de manera armonizada y eficiente para garantizar un empleo seguro y competitivo de los recursos, 
así como para suprimir todos los obstáculos que se interponen en el mercado interior del transporte, fomentar tecnologías limpias y modernizar las redes de transporte. El CESE pide que los reglamentos se adopten rápidamente y que en ellos se tengan en cuenta sus propuestas.

Al igual que en sus anteriores dictámenes, el CESE recomienda elaborar un modelo de internalización en el cálculo de $\mathrm{CO}_{2}$ de todas las emisiones relacionadas con la producción de automóviles. La huella del $\mathrm{CO}_{2}$ debería tenerse en cuenta porque afecta a todo el ciclo de vida de los automóviles.

K. Dictamen del Comité Económico y Social Europeo sobre "Río+20: balance y perspectivas" (Dictamen adicional) (2013/C 44/11)

El documento final de la Conferencia de las Naciones Unidas sobre el Desarrollo Sostenible (Conferencia Río+20), bajo el título de "El futuro que queremos", ha sido menos ambicioso de lo que el CESE hubiera deseado. En particular, no se ha prestado una adecuada atención a la urgencia de la situación de crisis que vive nuestro planeta. El documento final contiene, sin embargo, varios elementos que pueden servir de fundamento también para las acciones de la UE. Hay que destacar en especial el acuerdo global para conseguir que la "economía verde" sea un importante instrumento del desarrollo sostenible e incluya la dimensión social, así como el acuerdo para poner en marcha un proceso que conduzca a objetivos mundiales de desarrollo sostenible estrechamente ligados a los objetivos de desarrollo del milenio.

El CESE estima que el proceso posterior a Río+20 y la aplicación de las decisiones tomadas en la Conferencia deben incluir la participación de la sociedad civil. Por ello, acoge favorablemente y de forma expresa todos los esfuerzos realizados en este sentido por el resto de instituciones. El CESE seguirá fomentando, al igual que antes de la celebración de la Conferencia Río+20, el diálogo de la sociedad civil de la sostenibilidad, lo que también incluirá la participación de las organizaciones y redes europeas de la sociedad civil, así como de los consejos económicos y sociales y de los consejos de sostenibilidad nacionales.

De esta forma, el CESE participará activamente en la elaboración de un conjunto de objetivos mundiales de desarrollo sostenible fomentando, al igual que antes de la celebración de la Conferencia Río+20, el diálogo de la sociedad civil en la UE, pero 
también con nuestros socios de la sociedad civil de fuera de la Unión. En particular, intentará poner en contacto a las partes interesadas del proceso de objetivos de desarrollo sostenible, por una parte, con las del proceso de los objetivos del milenio, por otra. Además, sobre la base de su experiencia y composición, el CESE puede contribuir especialmente a dar forma concreta a la economía ecológica, incluida su dimensión social, y a los derechos de participación de la sociedad civil a escala mundial.

Acoge favorablemente las conclusiones del Consejo sobre Río+20, del 25 de octubre de 2012, que anuncian un ambicioso seguimiento de Río+20 - que se realizará a través de la Estrategia Europa 2020 y de la Estrategia de Desarrollo Sostenible de la UE—, así como la revisión programada de la Estrategia de Desarrollo Sostenible de la UE. El CESE considera necesario mantener un amplio debate de la sociedad civil sobre el desarrollo sostenible en la UE, y lo seguirá fomentando en sus futuros trabajos.

L. Dictamen del Comité Económico y Social Europeo sobre la "Propuesta de Decisión del Parlamento Europeo y del Consejo, sobre las normas contables y los planes de acción aplicables a las emisiones y absorciones de gases de efecto invernadero resultantes de las actividades enmarcadas en el uso de la tierra, el cambio de uso de la tierra y la silvicultura" [COM (2012) 93 final — 2012/0042 (COD), (2012/C 351/19)]

El Comité acoge favorablemente el texto de la Comisión ya que, en su opinión, se trata de una propuesta ambiciosa que responde a la necesidad de disponer de un sistema contable más riguroso, con el objeto de incorporar las recomendaciones de los acuerdos internacionales a la legislación de la UE. Cuando prepare y redacte actos normativos, la Comisión debería garantizar que los documentos pertinentes se transmitan al Parlamento Europeo, al Consejo y a la sociedad civil de forma simultánea, puntual y adecuada. La Comisión debería llevar a cabo las consultas apropiadas, también con expertos, en relación con la actualización de las definiciones en función de los cambios acordados por los órganos de la Convención Marco de las Naciones Unidas sobre el Cambio Climático (CMNUCC) o el Protocolo de Kioto, o de aquellas adoptadas con arreglo a otros acuerdos multilaterales. Resulta muy importante garantizar la compatibilidad de la propuesta con las decisiones adoptadas en el contexto de la CMNUCC.

Teniendo en cuenta que antes de 2015 se deberá negociar un acuerdo jurídico universal sobre el cambio climático que, según los planes actuales, entraría en vigor en 2020, la 
UE debería centrarse ahora en el desarrollo de unos modelos equitativos y eficaces, desde el punto de vista del clima, que fomenten la mitigación del cambio climático a fin de contribuir a las negociaciones sobre dicho acuerdo mundial. En este ámbito, el Land Use, Land-Use Change and Forestry (LULUCF) desempeña un papel clave, por lo que es importante disponer de normas comunes tanto sobre el cálculo de las emisiones como de los sumideros de emisiones.

M. Dictamen del Comité Económico y Social Europeo sobre la Comunicación de la Comisión al Parlamento Europeo, al Consejo, al Comité Económico y Social Europeo y al Comité de las Regiones "Energías renovables: principales protagonistas en el mercado europeo de la energía" [COM(2012) 271 final], (2013/C 44/24)

El CESE acoge favorablemente una comunicación que abre un debate necesario sobre la reformulación de las energías renovables y las correspondientes políticas. En opinión del Comité, un sistema de regímenes de ayuda común para toda la Unión Europea adaptado para cada tecnología podría responder mejor a las exigencias de eficiencia y del mercado interior. Los regímenes deberían tener una limitación temporal - hasta que las tecnologías sean competitivas-, no prever una compensación excesiva y garantizar un apoyo adaptado a las soluciones locales de pequeña escala. No obstante, mientras el mercado interior de la energía no funcione adecuadamente, los regímenes de ayuda deberían adaptarse a cada zona tarifaria de la electricidad o Estado miembro.

Las tecnologías en el sector de las fuentes de energía renovables ofrecen grandes oportunidades, al igual que otras tecnologías, para la reducción de los gases de efecto invernadero - carbón limpio, almacenamiento de electricidad, respuesta a la demanda, uso del carbono, fisión y fusión nucleares...- o para la reducción de otros gases de efecto invernadero como el metano, etc. En muchos casos los avances son claramente prometedores, por lo que deben incentivarse de manera adecuada. En particular, se deberían respaldar las demostraciones tecnológicas y la difusión rápida de las nuevas tecnologías.

En este sentido, el CESE recomienda a la Comisión que centre sus futuros trabajos para después de 2020 en una política orientada a la descarbonización. Finalmente, esta política podría renunciar a los objetivos para la energía renovable y, en lugar de ello, basarse en un claro objetivo de reducción de los gases de efecto invernadero, según las 
necesidades de reducción de los gases de efecto invernadero a largo plazo, y en un precio del carbono lo suficientemente alto como para que los operadores relevantes tomen medidas para mejorar la eficiencia y contribuyan a la I+D y las inversiones, pero no demasiado alto para los consumidores y la competitividad industrial. Se necesitan, además, medidas específicas para impulsar el desarrollo de las tecnologías en el sector de las fuentes de energía renovables y las inversiones en estas, que son las que, en definitiva, aportarán un cambio real. Lo ideal sería que estas medidas fueran comunes para toda la UE y adaptadas para cada tecnología. 